\title{
Ostéologie et systèmes humains
}

\author{
Giovanni Briganti
}

giovanni.briganti@hotmail.com 


\section{Méthodologies de l'étude anatomique}

Les trois principales façons d'aborder l'anatomie sont les approches régionale, systémique et clinique.

1. Anatomie régionale:

L'anatomie régionale étudie le corps région par région (comme par ex : le thorax, l'abdomen, la tête et le cou). Elle traite des rapports structuraux existant entre les différentes parties constitutives de la région concernée. L'ordinateur est un outil utile à l'enseignement de l'anatomie régionale. Il facilite la visualisation des images obtenues par tomodensitométrie (TDM) ou par résonnance magnétique (IRM) et la manipulation de figures anatomiques en 3D. L'expérience personnelle de la dissection permet également une excellente visualisation des parties du corps.

L'anatomie de surface en est un aspect essentiel. Elle permet d'étudier l'anatomie du sujet vivant au repos ou en activité. Le but de cette méthode est de visualiser les structures palpables situées sous la peau.

\section{Anatomie systémique :}

L'anatomie systémique est l'étude des systèmes corporels. Les différents systèmes sont les suivants :

- Système tégumentaire : comprend la peau et ses divers appendices (poils, ongles, glandes sudoripares). La peau est non seulement une enveloppe protectrice mais aussi un organe sensoriel étendu.

- Système squelettique : se compose d'os et de cartilages. Il forme le tissu de soutien du corps et protège certains organes vitaux. 
- Système articulaire : comprend les articulations et leurs ligaments. Il assure la connexion entre les différentes portions osseuses et représente l'ensemble des sites où se réalisent les mouvements.

- Système musculaire: se compose de muscles qui se contractent pour mobiliser les différentes parties du corps.

- La plupart des éléments des systèmes squelettique, articulaire et musculaire forment le système locomoteur.

- Système circulatoire: comprend les systèmes cardio vasculaire et lymphatique qui fonctionnent en parallèle. Le système cardio vasculaire comprend le cœur et les vaisseaux sanguins qui assurent la circulation du sang dans le corps. Le système lymphatique se compose d'un réseau de vaisseaux lymphatiques qui drainent l'excès de fluide tissulaire des compartiments intercellulaires du corps. Il participe également à la défense de l'organisme.

- Système nerveux : comprend le système nerveux central (encéphale + moelle épinière) et le système nerveux périphérique (nerfs crâniens et spinaux avec leurs terminaisons motrices et sensorielles). Il contrôle et coordonne les fonctions des organes et met le corps en relation avec son environnement.

- Système digestif : comprend tous les organes impliqués dans l'ingestion, la mastication, la déglutition, la digestion, l'absorption des aliments et l'élimination des déchets.

- Système respiratoire: comprend les voies aériennes et les poumons qui fournissent l'oxygène au corps et élimine le CO2.

- Système urinaire : comprend les reins qui filtrent le sang et produisent l'urine, les uretères qui la transportent, la vessie qui l'accumule et l'urètre qui l'élimine. 
- Système reproducteur : comprend les organes génitaux impliqués dans la reproduction.

- Système endocrinien: comprend des glandes dépourvues de canaux excréteurs qui produisent des hormones transportées par le sang vers toutes les parties du corps (ex: thyroïde).

\section{Anatomie clinique :}

L'anatomie clinique établit la corrélation avec la pratique médicale. Elle incorpore les approches régionale et systémique et souligne leurs applications cliniques. Les techniques d'endoscopie et d'imagerie contribuent à démontrer l'anatomie du vivant. 


\section{Terminologie anatomo-médicale}

L'anatomie et la médecine ont un vocabulaire international. Il existe une nomenclature scientifique qui permet une communication précise entre tous les professionnels de la santé de par le monde. Depuis 1976, il existe une nouvelle nomenclature anatomique conforme à la nomenclature latine internationale. Cette nouvelle terminologie voit la suppression de nombreux éponymes, c'est-à-dire des termes associant un nom propre (ex: angle de Louis remplacé par angle sternal dans la nouvelle terminologie). De plus, la terminologie anatomique introduit pour une large part la terminologie médicale.

\section{La position anatomique de référence}

Toutes les descriptions anatomiques se réfèrent à cette position anatomique afin d'éviter toute ambiguïté. La position anatomique est celle dans laquelle le sujet est debout, les pieds joints, la face, les yeux et les paumes des mains étant dirigés vers l'avant.

Cette position anatomique est utilisée universellement dans les descriptions anatomomédicales. Elle permet d'établir des relations entre n'importe quelle partie du corps et n'importe quelle autre partie.

\section{$\underline{\text { 2. Les plans anatomiques }}$}

Trois types de plans, perpendiculaires les uns aux autres, peuvent être définis chez un sujet étant en position anatomique.

2.1. Le plan médian : plan vertical passant longitudinalement à travers le corps le divisant en 2 moitiés gauche et droite. Les plans sagittaux: sont des plans verticaux parallèles au plan 
médian. Il est utile de donner un point de référence en nommant une structure traversée par le plan dont on parle (ex : plan sagittal passant par le milieu de la clavicule gauche).

2.2. Les plans coronaux ou frontaux : plans verticaux traversant le corps perpendiculairement par rapport au plan médian et le divisant en une partie antérieure (ventrale, avant) et une partie postérieure (dorsale, arrière).

2.3. Les plans horizontaux (transversaux): traversent le corps perpendiculairement par rapport aux plans médian et coronal. Il divise le corps en une partie supérieure (crâniale) et une partie inférieure (caudale). Il est utile de donner un point de référence pour identifier le niveau du plan (ex : plan horizontal passant par l'ombilic).

\section{Les bases de la terminologie anatomique}

3.1. Les termes utilisés dans l'étude des rapports et dans la description comparative:

Différents adjectifs (généralement regroupés en paires opposées) permettent de décrire les rapports entre des parties du corps et de comparer la position relative de 2 structures l'une par rapport à l'autre :

- superficiel (proche de la surface), intermédiaire (entre une structure superficielle et profonde), profond (éloigné de la surface)

- en avant = antérieur ou ventral

- en arrière $=$ postérieur ou dorsal

- en haut = supérieur ou crânial

- en bas = inférieur ou caudal

- près du plan médian = médial ou interne 
- à distance du plan médian = latéral ou externe

- proche de l'origine d'un membre = proximal ; loin = distal

3.2. Terminologie des mouvements :

Différents termes permettent de décrire les mouvements des membres ou d'autres parties du corps :

- Abduction : mouvement qui écarte un membre du plan médian

- Adduction : mouvement qui rapproche un membre du plan médian

- Flexion : mouvement vers l'avant d'une partie du corps par rapport au reste du corps

- Extension : mouvement qui amène cette même partie du corps vers l'arrière

- Pronation : mouvement de rotation interne de $180^{\circ}$ de la main

- Supination : mouvement inverse de la main

- Opposition : mouvement qui porte la pulpe du pouce vers la pulpe d'un autre doigt 


\section{Ostéologie}

\section{Introduction à l'ostéologie - Définitions}

Système squelettique : ensemble de structures rigides du corps contribuant à son maintien.

Os : Tissu conjonctif hautement spécialisé et rigide

Cartilage : Tissu conjonctif élastique et semi-rigide

Forme des os:

- Os longs : possèdent une partie centrale, appelée diaphyse, et deux extrémités, appelées épiphyses (proximale et distale).

- Os courts : cuboïdes. Se retrouvent dans le poignet et la cheville.

- Os irréguliers : possèdent formes variables, par exemple l'os coxal.

\section{Structure de l'os:}

- Os compact : l'os compact est très dense (très clair - blanc en radiographie). Il se trouve en périphérie de tous les os et entoure l'os spongieux.

- Os spongieux : est situé généralement à l'intérieur des os ; il possède des cavités.

Dans les diaphyses et à l'intérieur de l'os spongieux se trouve la cavité médullaire qui contient et produit la moelle osseuse, formée de cellules hématopoiétiques qui fabriquent les globules rouges.

- Périoste : enveloppe externe entourant l'os compact. Il intervient dans le cal osseux, réparation des os. Il est richement innervé et très sensible aux stimuli douloureux. 
- Diaphyse : la vascularisation se fait via des foramina nourriciers. Ce principe vasculaire est utilisé en chirurgie pour réaliser des transplantations osseuses libres appelées lambeaux libres, déplaçant une fraction d'os à un endroit différent du site de prélèvement, ainsi que des lambeaux pédiculés, déplaçant la fraction latéralement en gardant la même vascularisation.

- Epiphyse : se fait à partir de l'extérieur via des artères épiphysaires.

- Périoste : la vascularisation périostée se fait à travers la membrane.

\section{Développement de l'os}

- Ossification intra-membraneuse : se fait via du tissu conjonctif évoluant en tissu osseux.

- Ossification enchondrale : diffère de l'intra-membraneuse par une étape intermédiaire cartilagineuse. Elle se fait dans la diaphyse de manière centrifuge (du centre vers l'extérieur) et séparément dans les épiphyses. Une zone appelée métaphyse se trouve alors entre l'épiphyse et la métaphyse constituant le cartilage de croissance. Une lésion unilatérale de la métaphyse peut entraîner des retard de croissance de l'os peut mener à une anisomélie (différence de longueur entre deux os symétriques).

- Développement du crâne : pendant son développement, le crâne s'agrandit et unit les os qui à la naissance sont séparés (la séparation sert à permettre la croissance du cerveau). Les points de fusion entre deux os forment une suture. Les craniosténoses sont des pathologies de sous-développement du crâne.

Classification des os : l'humain possède 206 os, repartis en squelette axial et squelette appendiculaire. 
- Squelette axial : Le squelette axial comprend les os du crâne, de la colonne vertébrale, de la cage thoracique, et l'os hyoïde.

- Squelette appendiculaire: le squelette appendiculaire comprend tous les os des membres avec les ceintures respectives (scapulaire et lombaire).

\section{$\underline{1 . \text { Le crâne }}$}

Le crane forme le squelette de la tête. Il comprend 2 parties :

- Le neurocrâne (boite crânienne) qui renferme l'encéphale, les méninges et les nerfs crâniens. Il comprend une voute du crâne (ou calvaria) et un plancher (ou base du crâne)

- Le massif facial : constitue les orbites et comprend les os qui entourent les cavités orales ou nasales.

En position anatomique, le crâne est orienté de telle manière que le bord infra-orbitaire et le bord supérieur du méat acoustique externe (conduit auditif externe) se trouvent dans un plan horizontal : on parle de plan orbito-meatal (plan de Frankfort).

\subsection{Face antérieure}

Os frontal :

L'os frontal s'articule inféro-latéralement avec l'os zygomatique et inféro-médialement avec l'os nasal, la branche montante de l'os maxillaire, l'os lacrymal, ethmoïde et sphénoïde. On lui décrit une partie squameuse (lame verticale) et une partie horizontale (lame orbitaire) formant le toit de l'orbite et plancher de la cavité crânienne. Le bord supra-orbitaire présente trois éléments : la glabelle, protubérance centrale située au-dessus du nasion (point de rencontre 
entre la suture inter-nasale et l'os frontal), l'incisure frontale, latérale à la glabelle et rejoignant l'orbite oculaire, et le foramen supra-orbitaire, latéral et supérieur à l'incisure frontal et livrant passage aux vaisseaux et au nerf supra-orbitaire (branche du nerf V1 ophtalmique donnant la sensibilité du front ; le nerf ophtalmique provient du nerf $\mathrm{V}$ trijumeau donnant la sensibilité de la face et quelque branche motrice à destination des muscles du cou). Au-dessus du bord supra-orbitaire, on retrouve l'arcade sourcilière (correspondant au sourcil). L'os frontal présente le processus zygomatique de l'os frontal, ainsi qu'un processus maxillaire s'articulant avec la branche montante de l'os maxillaire.

\section{Os zygomatiques :}

Les os zygomatiques correspondent aux parties saillantes des joues. Ils s'articulent supérolatéralement avec l'os frontal et inféro-latéralement avec l'os maxillaire, le sphénoïde et le temporal. Ils présentent le processus frontal de l'os zygomatique. L'os zygomatique présente également un foramen zygomatico-facial par lequel passe le nerf zygomatico-facial, branche du nerf V2 maxillaire provenant du nerf V trijumeau.

Os nasaux :

Les os nasaux s'articulent crânialement avec l'os frontal par la suture fronto-nasale (le nasion étant l'union entre la suture inter-nasale et l'os frontal) et inféro-latéralement avec le maxillaire. Ils constituent le tiers supérieur du nez (appelé également la route montante du nez). Sous les os nasaux se trouve l'ouverture piriforme (ouverture antérieure des fosses nasales). A travers cette ouverture, on aperçoit le septum nasal osseux qui sépare la fosse nasale gauche de la droite : il est constitué d'une partie postéro-inférieure, le vomer, os se prolongeant vers la face inférieure du crâne postérieurement à l'os palatin, et une partie 
antéro-supérieure, la lame perpendiculaire de l'os ethmoïde, se prolongeant vers la face interne de la base du crâne par la cristagalli. La paroi latérale des fosses nasales porte des plaques osseuses incurvées : les cornets nasaux inférieurs, moyens et supérieurs.

Os maxillaires :

Les os maxillaires forment le squelette de la mâchoire supérieure et constitue le tiers moyen de la face antérieure du crâne. Leurs processus alvéolaires (bords alvéolaires) renferment les alvéoles dentaires (tissu de soutien pour les dents maxillaires, formant l'arcade dentaire supérieure). Ils entourent presque complètement l'ouverture piriforme et forment la partie médiale des bords infra-orbitaires. Ils présentent un orifice, le foramen infra-orbitaire qui livre passage aux vaisseaux et nerf infra-orbitaires, branche du nerf V2 maxillaire ( $2^{\text {ème }}$ branche du nerf $\mathrm{V}$ trijumeau) donnant la sensibilité de la joue.

\section{Orbite oculaire :}

L'orbite oculaire est la poche osseuse contenant le globe oculaire ainsi que les muscles, vaisseaux et nerfs assurant sa fonction. La paroi antérieure de l'orbite ou toit est constituée par la partie horizontale de l'os frontal. La paroi médiale est constituée supérieurement par l'os frontal, l'os maxillaire et lacrymal antérieurement, et l'os ethmoïde postérieurement. L'os lacrymal présente la crête lacrymale externe et interne (ou antérieure et postérieure), deux reliefs osseux séparés par une fosse lacrymale (ou sillon lacrymal) ou s'abouche le canal ou conduit lacrymal pour le larmoiement. La paroi latérale est constituée par l'os zygomatique antérieurement et par la grande aile de l'os sphénoïde (se prolongeant dans la paroi postérieure de l'orbite). La grande aile du sphénoïde présente la fissure orbitaire supérieure, foramen livrant passage aux nerfs III (oculomoteur commun), IV (trochléaire), VI 
(oculomoteur externe ou abducens), V1 (ophtalmique) et la veine ophtalmique supérieure. La veine ophtalmique inférieure passe dans le fissure orbitaire inférieure. La petite aile du sphénoïde, médiale à la grande aile du sphénoïde, présente le canal optique, foramen livrant passage au nerf II optique pour la vision ainsi qu'à l'artère ophtalmique innervant l'oeil.

\section{Mandibule :}

La mandibule est un os en forme de U. On la divise en une partie horizontale (corps) et une partie verticale (branche montante de la mandibule) se terminant par une tête surplombée par un condyle s'insérant dans la fosse mandibulaire de l'os temporal et participant à l'articulation temporo-mandibulaire. En avant de la tête se trouve le processus coronoïde. Son processus (ou bord) alvéolaire porte les dents mandibulaires constituant l'arcade dentaire inférieure. Endessous de la $2^{\text {ème }}$ prémolaire, on aperçoit de chaque côté le foramen mentonnier (livrant passage aux vaisseaux et au nerf mentonnier, branche du nerf V3 mandibulaire, branche du nerf $\mathrm{V}$ trijumeau, donnant la sensibilité de la mandibule). La mandibule présente une protubérance mentonnière qui forme le menton. Elle s'étend vers le bas à gauche et à droite par des reliefs appelés tubercules mentonniers.

\subsection{Face latérale}

Elle se compose antérieurement de l'os frontal qui s'articule postérieurement avec le pariétal et inférieurement avec le sphénoïde. On décrit une fosse temporale limitée en haut et en arrière par les lignes temporales, en avant par l'os frontal et zygomatique, en bas par l'arcade zygomatique : la fosse temporale sert d'insertion au muscle temporal. L'arcade zygomatique est formée par l'union du processus zygomatique du temporal et le processus temporal du zygomatique. Le ptérion est une jonction osseuse correspondant à une suture en forme de $\mathrm{H}$ 
unissant les os frontal, pariétal, sphénoïde et temporal. L'os temporal présente une partie supéro-antérieure squameuse de l'os temporal (écaille du temporal) correspondant à la fosse temporale et une partie postéro-inférieure mastoïdienne. En avant de celle-ci, on trouve l'ouverture acoustique externe qui est l'entrée du méat acoustique externe (conduit auditif externe). Le processus mastoïde est situé en arrière et en dessous de l'ouverture acoustique externe. Ventralement et médialement par rapport au processus mastoïde se trouve le processus styloïde. Entre ces deux processus, sur la face inférieure on pourra observer le foramen stylomastoïdien livrant passage au nerf facial VII. Enfin, on retrouve la mandibule qui se compose d'une partie horizontale (corps) et d'une partie verticale (branche montante de la mandibule).

\subsection{Face postérieure (ou occiput)}

La face postérieure est de forme ovoïde et est formée par l'os occipital, partie postérieure des os pariétaux et la partie mastoïdienne des os temporaux. Il existe une saillie dans le plan médian : la protubérance occipitale externe (inion). Celle-ci est reliée au foramen magnum par la crête occipitale externe. La ligne nucale supérieure prolonge latéralement la protubérance occipitale externe. Au centre de l'occiput, le lambda marque le point de rencontre des sutures sagittale et lambdoïde.

\subsection{Face supérieure}

Différents os sont apparents : l'os frontal en avant, les os pariétaux sur les côtés et l'os occipital en arrière. La suture coronale sépare l'os frontal des os pariétaux. La suture sagittale sépare les 2 os pariétaux. La suture lambdoïde sépare les os pariétaux et temporaux de l'os occipital. Bregma est le point de rencontre des sutures coronale et sagittale. Lambda est le point de 
rencontre des sutures sagittale et lambdoïde. Le vertex est le point culminant du crâne qui se situe +/- au milieu de la suture sagittale.

1.5. Face externe de la base du crâne

Os maxillaire et os palatin:

On y retrouve le palais dur (palais osseux) formé en avant par les processus palatins des os maxillaires et en arrière par les lames horizontales des os palatins. Sur la ligne médiane de l'os maxillaire se trouve la fosse incisive est une dépression située derrière les incisives centrales qui livre passage aux nerfs naso-palatins (branche du nerf V2 maxillaire). Les grands et petits foramina palatins s'ouvrent au niveau de la partie postéro-latérale du palais osseux. Les choanes (ouverture postérieure des fosses nasales) sont séparées par le vomer.

Os sphénoïde :

L'os sphénoïde est enchâssé entre les os frontal, temporaux et occipital. Il présente un corps et 3 paires de processus : grandes ailes et petites ailes (expansions latérales du corps) ainsi que les processus ptérygoïdes (expansions inférieures de la zone de jonction entre le corps et la grande aile). Chaque processus ptérygoïde se compose d'une lame latérale et une lame médiale, séparées par une fosse ptérygoïdienne. L'os sphénoïde présente les foramina en croissant, mieux visibles sur la face interne du crâne ; néanmoins, le foramen oval (livrant passage au nerf V3 mandibulaire) ainsi que l'épineux (livrant passage à une petite branche du V3 et des vaisseaux méningées) sont bien visibles sur la face inférieure du crâne.

Os temporal :

L'os temporal est creusé d'une dépression, la fosse mandibulaire (ou cavité glénoïde du temporal) dans laquelle vient se loger le condyle de la mandibule lorsque la bouche est fermée. 
L'os temporal présente sur sa face inférieure différents foramina: médialement et supérieurement, de chaque côté de la partie basilaire de l'os occipital, se trouve le foramen déchiré ou lacerum, rempli de cartilage sur le vivant. Inféro latéralement au foramen déchiré et supéro médialement au processus styloïde se trouve le canal ou foramen carotidien, livrant passage à l'artère carotide interne. Inférieurement au canal carotidien, confinant avec l'os occipital, se trouve le foramen jugulaire, livrant passage à la veine jugulaire interne ainsi que les nerfs crâniens IX glossopharyngien (contribuant à l'innervation de la langue et du pharynx), $X$ vague (système parasympathique ainsi qu'innervation du larynx), et XI spinal ou accessoire (innervation motrice du trapèze et sternocléidomastoïdien). Latéralement au foramen jugulaire, derrière le processus stylö̈de, se trouve le foramen stylomastö̈dien livrant passage au nerf VII facial pour l'innervation motrice des muscles de la face.

Os occipital :

L'os occipital s'articule en avant avec l'os sphénoïde. Il entoure le foramen magnum par où passent la moelle épinière et ses enveloppes, les artères vertébrales et les artères spinales antérieures et postérieures. La partie basilaire (= clivus à la face interne de la base du crâne) est le prolongement entre les 2 os temporaux et s'articule avec le sphénoïde en avant. Ventralement et latéralement par rapport au foramen magnum, on observe deux reliefs osseux importants: les condyles occipitaux par lesquels le crane s'articule avec la $1^{\text {ère }}$ vertèbre cervicale atlas). En avant du condyle occipital et derrière le foramen jugulaire se trouve le canal du nerf hypoglosse, se prolongeant dans la face interne de la base du crâne. Derrière le condyle occipital se trouve le canal condylaire, livrant passage à une veine émissaire.

1.6. Face interne de la base du crâne 
La face interne de la base du crâne comprend 3 grandes dépressions qui correspondent à 3 niveaux différents : les fosses crâniennes antérieure, moyenne et postérieure.

a. Fosse crânienne antérieure

La fosse crânienne antérieure est formée par l'os frontal en avant, l'os ethmoïde médialement et le corps et petites ailes de l'os sphénoïde postérieurement.

Os frontal :

La plus grande partie de cette fosse est occupée par la partie orbitaire de l'os frontal. Cette partie porte les lobes frontaux et constitue le toit de l'orbite. Il existe une saillie osseuse médiane de l'os frontal : la crête frontale. A sa base se trouve le foramen caecum.

Os ethmoïde :

La cristagalli est une crête osseuse médiane appartenant à l'os ethmoïde et située en arrière du foramen caecum. De chaque côté de la cristagalli se trouve la lame criblée de l'os ethmoïde. Cette dernière a l'aspect d'une passoire et livre passage à des radicelles nerveuses du nerf I. Toujours dans la lame criblée, se trouvent les foramina ethmoïdaux antérieurs et postérieurs livrant passage à des branches homonymes du V1 et se prolongeant dans la partie ethmoïdale de l'orbite oculaire.

Os sphénoïde :

Les petites ailes de l'os sphénoïde présentent un prolongement médian et postérieur : les processus clinoïdes antérieurs. Entre les 2 petites ailes se trouve le limbus sphénoïdal.

b. Fosse crânienne moyenne 
Sa forme évoque celle d'un papillon. Elle présente un relief en forme de selle, la selle turcique, située à la face sup du corps de l'os sphénoïde. Celle-ci est flanquée de 2 dépressions latérales où viendront se loger les lobes temporaux du cerveau. Ces dépressions latérales sont constituées ventro-médialement par les grandes ailes de l'os sphénoïde, ventrolatéralement par l'écaille du temporal (partie squameuse) et dorsalement par le rocher (partie pétreuse du temporal). Les limites de la fosse crânienne moyennes sont antéro-latéralement le bord tranchant des petites ailes de l'os sphénoïde, antéro-médialement le limbus sphénoïdal (bord antérieur du sillon pré chiasmatique, lieu de réunion entre les deux nerfs optiques, se séparant en suite par deux bandelettes optiques, une gauche et une droite, allant dans le cortex occipital pour délivrer l'information visuelle), postéro-latéralement la partie pétreuse des os temporaux et postéro-médialement le dorsum sellae.

La selle turcique est bordée par les processus clinoïdes antérieurs et postérieurs et comprend 3 parties: le tubercule de la selle (situé en avant de la fosse hypophysaire), la fosse hypophysaire où se loge l'hypophyse et le dos de la selle (dorsum sellae).

De chaque côté de la base du corps de l'os sphénoïde, 4 foramina disposés en croissant traversent la grande aile du sphénoïde : fissure orbitaire supérieure, foramen rond, foramen ovale, foramen épineux. La fissure orbitaire supérieure sépare la grande de la petite aile. Elle établit une communication entre l'orbite et la fosse crânienne moyenne. Elle livre passage aux nerf crâniens oculomoteurs III, IV, VI, le nerf ophtalmique V1 et à la veine ophtalmique supérieure. Le foramen rond est situé dorsalement par rapport à l'extrémité médiale de la fissure orbitaire supérieure. Il livre passage au nerf V2 maxillaire (sensibilité de la face). Le foramen ovale est plus grand que le foramen rond et se positionne postéro-latéralement par rapport à celui-ci. Il s'ouvre dans la fosse infra temporale et livre passage au nerf V3 
mandibulaire donnant la sensibilité de la mandibule ainsi que l'innervation motrice des muscles sus-hyoïdiens. Le foramen épineux se situe en arrière et latéralement par rapport au foramen ovale. Il livre passage à une petite branche du V3 réservée aux méninges et des artères méningées moyennes.

L'os temporal contribue à la fosse moyenne, présentant le méat acoustique interne situé audessus du foramen déchiré. Il livre passage au nerf VII vestibulo-cochléaire ou acoustique.

De part et d'autre du dorsum sellae et situé postéro-latéralement par rapport à la selle turcique se trouve le foramen déchiré. Ce dernier est obturé par une lame cartilagineuse. L'artère carotide interne passe à la face supérieure de ce foramen.

c. Fosse crânienne postérieure

Il s'agit de la plus grande et la plus profonde des 3 fosses crâniennes. Elle est principalement bordée par l'os occipital. Ce dernier possède un plan osseux incliné, le clivus. Postérieurement se trouve la crête occipitale interne qui aboutit à la protubérance occipitale interne. De part et d'autre de la crête occipitale interne se trouvent les fosses cérébelleuses qui contiennent le cervelet. Inférieurement au foramen déchiré se trouve le canal du nerf hypoglosse donnant la motricité de la langue.

\section{La colonne vertébrale}

\subsection{Introduction}

La longueur de la colonne vertébrale est en moyenne de $75 \mathrm{~cm}$ dont $1 / 4$ est constitué par les disques intervertébraux fibro-cartilagineux. Il existe un axe vertical présent à cause des 4 courbures (cervicale, thoracique, lombaire et sacrée) ayant des convexités différentes qui vont s'annuler. L'axe vertical est donc la superposition de ces 4 courbures. Les courbures cervicales 
et lombaires sont concaves vers l'arrière et convexes vers l'avant. Les courbures thoracique et sacrale sont concaves vers l'avant. Les courbures et la dégénérescence progressive des disques intervertébraux s'accentuent avec l'âge, causant une perte de taille. Il existe des déformations anormales :

- Accentuation de la courbure thoracique (dos voûté, bossu) = cyphose

- Accentuation de la courbure thoracique (dos cambré, creux $)=$ lordose

- Courbure latérale anormale avec rotation des vertèbres = scoliose

La colonne vertébrale est constituée de 2 sortes de vertèbres : 24 vertèbres mobiles $(7$ vertèbres cervicales, 12 thoraciques, 5 lombaires) avec les disques intervertébraux fibro-cartilagineux qui permettent la mobilité relative d'une vertèbre à l'autre et 9 vertèbres soudées ( 5 sacrales, 4 coccygiennes). Il est important de noter que la moelle épinière ne descend pas jusqu'à l'extrémité du coccyx : elle se termine inférieurement au niveau de la deuxième vertèbre lombaire L2.

\subsection{Vertèbre type}

Une vertèbre type (ou schématique) contient un corps vertébral (ou spondyle) qui est la partie antérieure de forme cylindrique et massive de la vertèbre. Postérieurement se détache l'arc neural (ou vertébral) qui délimite avec le corps : le foramen vertébral (laissant passer la moelle épinière jusque L1-L2). L'arc neural comprend des pédicules vertébraux gauches et droits unissant l'arc vertébral au corps vertébral ainsi que des lames en arrière qui s'unissent pour former le processus épineux. A l'union des pédicules et des lames se détachent latéralement vers l'arrière les processus transverses mais aussi 4 processus articulaires : 2 supérieurs (en haut et en arrière) et 2 inférieurs (en bas et en avant). Les processus articulaires inférieur et 
supérieur vont s'articuler avec les processus articulaires des autres vertèbres et stabilisent la colonne vertébrale pour empêcher les mouvements antéropostérieurs.

\subsection{Caractéristiques régionales des vertèbres}

-vertèbres cervicales typiques:

Le corps vertébral est petit et de forme rectangulaire. Il présente latéralement des processus semi-lunaires. Le foramen vertébral est triangulaire. Les processus transverses sont bituberculé et perforés par des foramina transversaires, où passent l'artère vertébrale (branche de l'artère sous-clavière, les plexus veineux et sympathiques). Le processus épineux est court et bifide. Les processus articulaires supérieurs sont orientés vers le haut et l'arrière tandis que les processus articulaires inférieurs sont orientés vers le bas et l'avant.

-vertèbres cervicales atypiques:

L'atlas (C1) ne présente pas de corps vertébral ni de processus épineux. On lui décrit 2 masses latérales réunies par les arcs antérieur et arc postérieur, délimitent un large foramen vertébral. Les masses latérales présentent deux surfaces ou fossettes articulaires: 2 supérieures (s'articulent avec les condyles occipitaux à la base externe du crâne) et 2 inférieures (presque planes et circulaires). Les processus transverses sont courts et creusés par les foramina transversaires. Au centre de la vertèbre on peut observer le ligament transverse séparant le canal vertébral en deux parties : la pars dentis antérieure réservée au passage de la dent de l'axis (permettant la rotation de la tête), et la pars nervosa postérieure livrant le passage à la moelle épinière.

L'axis (C2) présente un processus qui se détache verticalement au niveau du corps vertébral : la dent de l'axis (ou apophyse odontoïde). La dent est maintenue par un ligament transverse 
de l'atlas et joue un rôle de pivot autour duquel tournent l'atlas et la tête. Les processus articulaires ne sont pas placés les uns au-dessus des autres (les supérieurs sont autour de la dent et les inférieurs sont sous les lames). Les processus transverses sont courts et creusés par les foramina transversaires.

-vertèbres thoraciques:

Le point d'appui avec les cotes est la caractéristique principale des vertèbres thoraciques. Le corps vertébral est aplati dans le sens de la hauteur et présente des facettes costales supérieures pour l'articulation avec la tête costale. La tête costale s'appuie sur la vertèbre de même niveau et celle du niveau inférieur (exemple: $5^{\text {ème }}$ côte s'appuie sur T5 et T6). Les processus transverses présentent des facettes costales transversaires pour les articulations avec le tubercule costal. Le foramen est plus petit et circulaire. Le processus épineux est long et incliné vers le bas. Les processus articulaires supérieurs regardent en arrière et latéralement et les processus articulaires inférieurs regardent en avant et médialement.

-vertèbres lombaires :

Le corps vertébral est massif et le foramen vertébral est triangulaire. Les processus transverses sont longs et grêles. Le processus épineux est court, robuste, en forme de hachette. Les processus articulaires supérieurs regardent médialement et les processus articulaires inférieurs regardent latéralement.

\section{Le squelette thoracique}

\subsection{Généralités}

Le squelette thoracique est composé par le sternum et 12 paires de côtes. La totalité des côtes n'est pas attachée au sternum : les côtes 11 et 12 sont flottantes, les côtes 8,9 et 10 ne s'attachent 
pas directement au sternum mais bien au cartilage costal de la côte 7 ; elles sont appelées des fausses côtes, puisque le cartilage costal fait la jonction directe entre une vraie côte et le sternum, sa présence permet une plus grande mobilité lors de la respiration.

\subsection{Le sternum}

Le sternum est un os plat, allongé, légèrement incurvé vers l'avant et situé au milieu de la cage thoracique. Il mesure 15 à $20 \mathrm{~cm}$ de long et 5 à $6 \mathrm{~cm}$ de largeur. Il est composé de 3 parties : le manubrium, le corps et le processus xiphoïde. Le manubrium est situé +/- à hauteur de T3 et T4. Il présente latéralement deux incisures claviculaires (articulation avec la clavicule et la 1 ère cote). Entre les 2 incisures claviculaires se trouve l'incisure jugulaire. Les bords latéraux du

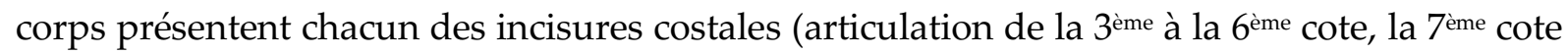
étant à cheval entre le corps et le processus xiphoïde). Entre le manubrium et le corps, il existe un angle: l'angle sternal (ou angle de Louis). Cet angle est palpable et correspond au $2^{\text {ème }}$ cartilage costal et sert donc de référence pour compter les cotes.

\subsection{Les cotes}

Les cotes mettent en relation le sternum en avant et la colonne vertébrale en arrière. Il y a 3 types de cotes :

- Les vraies cotes (1 à 7) sont unies au sternum par les cartilages costaux.

- Les fausses cotes (8 à 10) sont unies au cartilage costal sus-jacent (côte 7).

- Les cotes flottantes (11 et 12) ont leur extrémité libre.

La cote typique présente au niveau de l'extrémité dorsale une tête qui s'articule avec deux corps vertébraux et porte donc 2 facettes articulaires (supérieure et inférieure) séparées par 
une crête. La tête est réunie au corps par une portion plus rétrécie, le col. À l'union du corps et du col, on retrouve un renflement, le tubercule costal dont la partie caudale porte une facette articulaire pour le sommet du processus transverse de la vertèbre correspondante. Plus latéralement par rapport au tubercule, le corps change de direction en formant un coude: l'angle costal. Le corps de la cote présente une face externe convexe et une face interne concave. Le bord crânial est arrondi et le bord caudal est mince et tranchant. Le long du bord caudal, la face interne est creusée par la gouttière costale où passent cranio-caudalement la veine, artère et nerf intercostal (moyen mnémotechnique = VAN).

Il existe 3 cotes atypiques. La $1^{\text {ère }}$ cote est courte, large et angulée à angle droit. L'extrémité dorsale présente une tubérosité costale volumineuse. Au niveau de la face crâniale se trouve le tubercule du muscle scalène antérieur (Lisfranc) qui sépare la gouttière ventrale (veine subclavière) de la gouttière dorsale (artère subclavière).

Les 11 et $12^{\text {ème }}$ cotes n'ont pas de facettes articulaires pour les processus transverses des vertèbres thoraciques. La tête porte une seule facette articulaire.

\subsection{L'os hyoïde}

L'os hyoïde est suspendu par des muscles sus-hyoïdiens et sous-hyoïdiens entre la mandibule et le larynx. Il est en forme de U, présentant une grande corne et une petite corne latérosupérieurement se détachant de la partie centrale, le corps.

\section{Squelette du membre supérieur}

Le membre supérieur comprend 4 segments :

- La ceinture scapulaire (composée en avant par la clavicule et en arrière par la scapula) 
- Le bras (reliant l'épaule au coude et représenté par l'humérus)

- L'avant-bras (reliant le coude au poignet et représenté par l'ulna et le radius)

- La main (prolongement distal de l'avant-bras composé des carpes, métacarpes et phalanges).

\subsection{La clavicule}

La clavicule est un os doublement incurvé en S allongé qui est articulé médialement avec le sternum via l'articulation sternoclaviculaire, et latéralement avec la scapula via l'articulation acromio-claviculaire. Ses $2 / 3$ médiaux sont convexes et son 1/3 latéral est concave. La face crâniale ne présente pas de relief particulier. Elle est recouverte par la peau et le platysma, muscle cervical superficiel situé postérieurement à la graisse sous-cutanée cervicale. La face caudale présente le sillon du muscle subclavier avec l'insertion de ce muscle. Médialement se trouve une surface rugueuse pour l'insertion du ligament costo-claviculaire. Latéralement se trouve le tubercule conoïde (insertion du ligament conoïde) et la crête ou ligne trapézoïde (insertion du ligament trapézoïde). Le bord ventral donne l'insertion au muscle grand pectoral médialement et deltoïde latéralement. Le bord dorsal donne l'insertion au muscle sternocléido-mastoïdien médialement et trapèze latéralement. La clavicule présente également des facettes articulaires pour les 2 extrémités sternale et acromiale.

\subsection{La scapula}

La scapula est un os plat, triangulaire appliqué sur la face postéro-latérale du thorax en regard de la $2^{\text {ème }}$ à la $7^{\text {ème }}$ cote. La face dorsale est convexe et divisée en 2 parties inégales par un relief osseux appelé épine scapulaire (relief se dirigeant antérieurement et médialement) : fosse 
supra et infra épineuse remplies par les muscles supra et infra épineuse. L'extrémité latérale de l'épine scapulaire présente un élargissement aplati : l'acromion. La face ventrale est concave et excavée par la fosse subscapulaire (insertion du muscle subscapulaire). Le bord crânial présente à son extrémité latérale un relief osseux dirigé vers l'avant en forme de bec de corbeau = processus coracoïde. De part et d'autre du processus coracoïde se trouve latéralement le tubercule supra-glénoïdien et médialement l'incisure scapulaire. Le bord médial (ou spinal) est séparé de $5 \mathrm{~cm}$ des processus épineux des vertèbres thoraciques. Il donne l'insertion aux muscles élévateur de la scapula, muscle rhomboïde et muscle dentelé antérieur. Le bord latéral présente une surface articulaire pour la tête de l'humérus, la cavité glénoödale. Celle-ci est surmontée d'un tubercule supra et infra-glénoïdien où les muscles vont venir s'insérer.

\subsection{L'humérus}

L'humérus s'articule supéro-médialement avec la scapula au niveau de l'articulation scapulohumérale (gléno-humérale) et inférieurement avec l'ulna et le radius au niveau de l'articulation du coude.

\section{Epiphyse proximale :}

Elle comprend la tête qui s'emboite au niveau de la cavité glénoïdale de la scapula. Elle représente $1 / 3$ de la sphère et est $2 x$ plus étendue que la cavité glénoïdale. Le pourtour de la tête humérale est délimité par le col anatomique (jonction entre la surface articulaire et la surface non articulaire) qui sépare 2 tubérosités : tubercule majeur (latéralement) et tubercule mineur (médialement). Entre les 2 tubercules se trouve le sillon inter-tuberculaire livrant passage au tendon bicipital. L'extrémité proximale est liée au corps par le col chirurgical (site fréquent de fracture). 


\section{Diaphyse :}

Le corps de l'humérus présente latéralement sur la face ventrale une tubérosité deltoïdienne (insertion du muscle deltoïde). Le sillon du nerf radial parcourt obliquement la face dorsale où chemine le nerf radial.

\section{Epiphyse distale :}

L'épiphyse distale s'élargit en formant les crêtes supracondylaire médiale et latérale qui se terminent par les épicondyles médial et latéral. Entre ces 2 saillies, il existe une surface articulaire qui est divisée en deux parties inégales par une rainure longitudinale : surface articulaire en forme de poulie (médialement), la trochlée humérale qui s'articule avec l'incisure trochléaire de l'ulna et une surface articulaire arrondie (latéralement), le capitulum qui s'articule avec la tête du radius. Au-dessus de la trochlée se trouve la fosse coronoïdienne sur la face ventrale et la fosse olécrânienne sur la face dorsale. La première reçoit le processus coronoïde de l'ulna lors de la flexion complète. La seconde héberge l'olécrâne lors de l'extension complète du coude. Au-dessus du capitulum se trouve la fosse radiale sur la face ventrale qui reçoit le bord de la tête du radius lors de la flexion complète.

\subsection{L'ulna (ou cubitus)}

Il forme avec le radius le squelette de l'avant-bras.

\section{Epiphyse proximale :}

L'épiphyse proximale de l'ulna présente une excroissance antérieure le processus coronoïde et postérieure l'olécrâne. La face antérieure de l'olécrâne forme la paroi postérieure de l'incisure trochléaire qui s'articule avec la trochlée de l'humérus). Sur la face latérale du processus coronoïde se trouve l'incisure radiale de l'ulna qui s'articule avec la tête du radius. 
Sous le processus coronoïde se trouve la tubérosité de l'ulna correspondant au site d'insertion du tendon du muscle brachial.

Diaphyse distale :

Le corps de l'ulna est triangulaire dans sa partie proximale puis diminue en diamètre et devient cylindrique.

Epiphyse distale :

L'épiphyse distale s'élargit pour former une tête discoïdale et une excroissance conique médialement, le processus styloïde.

\subsection{Le radius}

Epiphyse proximale :

L'épiphyse proximale présente une tête, un col et une tubérosité. La tête est un petit cylindre dont le sommet est creusé en cupule pour s'articuler avec le capitulum huméral. Elle s'articule aussi par son pourtour avec l'incisure radiale de l'ulna. La tête est donc entièrement revêtue de cartilage. La tête est réunie au corps par une portion rétrécie, le col. La tubérosité bicipitale (insertion du muscle biceps) sépare la tête et le col du corps.

Diaphyse distale :

La face latérale du corps du radius est convexe. Il augmente graduellement de volume vers le poignet et est presque rectangulaire en coupe transversale à son extrémité distale. Le bord médial donne insertion à la membrane interosseuse.

Epiphyse distale : 
L'épiphyse distale présente médialement une surface articulaire pour la tête de l'ulna: l'incisure ulnaire du radius. Elle présente latéralement un prolongement, le processus stylö̈de. On observe dorsalement des sillons destinés au passage de tendons des muscles de l'avantbras.

4.6. Les os de la main

Le carpe:

Le carpe est composé de 8 os répartis en 2 rangées (dans l'ordre latéro-médial) :

- 1 ère rangée proximale :

○ Os scaphoïde : a la forme d'une petite barque. Il s'articule proximalement avec le radius et distalement avec le trapèze et trapézoïde. Il présente un tubercule saillant au niveau palmaire.

- Os lunatum : os semi-lunaire. Il s'articule proximalement avec le radius et distalement avec le capitatum et l'hamatum.

- Os triquetrum: os pyramidal. Il présente au niveau palmaire une facette articulaire pour l'os pisiforme. Il s'articule proximalement avec le radius et l'ulna ; distalement avec l'hamatum.

- Os pisiforme : a la forme d'un petit pois et est situé sur la face palmaire de l'os triquetrum.

- 2ème rangée distale :

- Os trapèze : porte sur sa face palmaire le tubercule du trapèze. Il s'articule proximalement avec le scaphoïde et distalement avec le 1 ${ }^{\text {er }}$ métacarpien. 
Os trapézoïde : en forme de coin. Il s'articule proximalement avec le scaphoïde et distalement avec le $2^{\text {ème }}$ métacarpien.

- Os capitatum (grand os) : a une tête arrondie. Il s'articule proximalement avec le lunatum et distalement avec le $3^{\text {ème }}$ et une partie du $4^{\text {ème }}$ métacarpien.

- Os hamatum (os crochu) : a la forme d'un coin et porte un processus crochu : le crochet ou hamulus. Il s'articule proximalement avec le triquetrum et distalement avec les $4{ }^{\text {ème }}$ et $5^{\text {ème }}$ métacarpiens.

L'ensemble des os du carpe a la forme d'une gouttière (U) ouvert en direction palmaire qui constitue le canal carpien. Les versants sont constitués du côté radial par les tubercules du scaphoïde et du trapèze et du coté ulnaire par le pisiforme et l'hamulus de l'os crochu.

Le métacarpe:

Entre le carpe et les phalanges se trouvent 5 métacarpiens. Chaque métacarpien possède un corps et 2 extrémités : proximale (en contact avec les os du carpe) et distale (en contact avec les phalanges proximales).

- $1^{\text {er }}$ métacarpien : est le plus large et le plus court. Il est en contact avec l'os trapèze.

- 2ème métacarpien : est en contact avec l'os trapézoïde,

- $\quad 3^{\text {ème }}$ métacarpien : présente un processus styloïde et est en contact avec le capitatum

- $4^{\text {ème }}$ métacarpien et $5^{\text {ème }}$ métacarpien : sont en contact avec l'os hamatum.

Les phalanges :

Chaque doigt comprend 3 phalanges (proximale - moyenne- distale) excepté le pouce qui n'en a que 2. Chaque phalange possède un corps et 2 extrémités. 


\section{$\underline{5 .}$ Ceinture pelvienne (bassin)}

Le bassin constitue 3 pièces osseuses. Il y a un os coxal droit et gauche. Ces 2 os coxaux s'unissent antérieurement sur la ligne médiale au niveau de la ceinture pelvienne et proximalement avec le sacrum.

\subsection{L'os coxal}

L'os coxal est constitué de 3 trois os distinct chez l'enfant qui fusionnent au cours de la croissance (ilium, pubis, ischium).

\section{Face externe :}

La face externe est marquée par la surface fessière ou glutéale (insertion des muscles fessiers) divisées par 3 lignes glutéales antérieures et qui constitue le relief des fesses. Au-dessus de cette surface se trouve la crête iliaque du bassin (hanche). Cette crête présente des lèvres externes et internes. En-dessous de cette surface se trouve une cavité avec une surface articulaire en demi-lune qui reçoit la tête du fémur, l'acétabulum. Celui-ci est à cheval sur l'ilium, pubis, ischium. En-dessous de l'acétabulum se trouve un trou, le foramen obturé. Ce foramen sépare la cavité pelvienne avec les organes génitaux. Il est délimité antérieurement par le corps du pubis où se détache supérieurement une branche montante et inférieurement une branche descendante, et postérieurement par l'ischium qui présente une branche inférieure se soudant avec la branche inférieure du pubis pour constituer la branche ischiopubienne.

Face interne :

La face interne présente de la ligne arquée étant une ligne oblique vers le bas et vers l'avant qui sépare l'os iliaque. Le bassin est divisé en 2 parties (grand et petit bassin). La limite entre 
ces 2 parties est le détroit supérieur. Ce plan passe par le bord supérieur de la ceinture pelvienne, par la ligne arquée et par le promontoire postérieur. La ligne subdivise cet os en 2 parties : supérieure et antérieure, la fosse iliaque légèrement excavée où s'insère le muscle iliaque ; postérieure : de haut en bas : la tubérosité iliaque (relief osseux avec des cavités qui sont des sites d'insertion de ligament). En-dessous de cette tubérosité iliaque, il y a une surface auriculaire pour l'articulation avec le sacrum. Il s'agit de l'articulation sacro-iliaque. Endessous de cette surface auriculaire se trouve le corps de l'ischium : foramen obturé.

\section{Bord supérieur :}

Le bord supérieur est marqué par la présence de la crête iliaque qui va de l'épine iliaque antéro-supérieure à l'épine iliaque postéro-supérieure.

\section{Bord inférieur :}

Ce bord présente un relief osseux, la tubérosité ischiatique.

\section{Bord antérieur :}

Ce bord présente 3 reliefs de haut en bas (épine iliaque antéro-supérieure, épine iliaque antéroinférieure et l'éminence ilio-pectinée). Au niveau de la branche supérieure du pubis se trouve une crête osseuse tranchante, le pecten du pubis.

\section{Bord postérieur :}

C'est de par ce bord postérieur que communique le petit bassin et le membre inférieur. La plupart des nerfs passent par la grande et petite échancrure sciatique. C'est dans la grande échancrure sciatique que passe le nerf sciatique. L'épine postéro-inférieure et postérosupérieure délimite ces échancrures. La grande échancrure sciatique se situe entre l'épine 
iliaque postéro inférieure et l'épine sciatique. La petite échancrure sciatique se situe entre l'épine sciatique et la tubérosité ischiatique.

\subsection{Le sacrum}

Le sacrum s'articule avec la surface auriculaire de l'os coxal et constitue l'articulation sacroiliaque. Le sacrum a une forme de pelle dont la concavité est tournée vers le bas et vers l'avant.

\section{Face antérieure :}

La face antérieure présente un trou qui livre passage aux branches du plexus sacré, les 4 foramina sacraux pelviens antérieurs.

\section{Face postérieure :}

La face postérieure présente une crête sacrale médiane constituée par le relief des processus épineux, une crête sacrale intermédiaire correspondant aux résidus des processus articulaires et une crête sacrale latérale correspondant aux résidus des processus transverses.

\section{Bord antérieur :}

Il fait protrusion dans le bassin. On parle de promontoire.

\section{$\underline{\text { 6. Squelette du membre inférieur }}$}

\subsection{Le fémur}

Le fémur est l'os le plus long $(+/ 45 \mathrm{~cm})$ et le plus lourd du corps.

\section{Epiphyse proximale :}

La tête fémorale est orientée vers le haut, médialement et vers l'avant. Elle s'articule avec l'acétabulum de l'os coxal. Elle est reliée à la diaphyse par le col du fémur. L'angle entre la tête 
et le corps est de $126^{\circ}$ en moyenne. A la jonction entre le col et la diaphyse, le fémur présente 2 trochanters: le grand trochanter (latéralement et crânialement) et le petit trochanter (médialement). Ces 2 trochanters se rejoignent par la ligne intertrochantérique sur la face ventrale et la crête intertrochantérique sur la face dorsale. Cette crête présente un renflement arrondi, le tubercule du muscle carré fémoral.

\section{Diaphyse :}

La face ventrale du corps du fémur ne présente pas de relief particulier. La face dorsale présente une arête rugueuse verticale, la ligne âpre qui présente au niveau du $1 / 3$ moyen du corps 2 lèvres : une lèvre médiale (qui se poursuit avec la ligne spirale en haut et la ligne supracondylaire en bas) et une lèvre latérale (qui se poursuit avec la tubérosité glutéale en haut et la ligne supracondylaire en bas). Une crête du pectiné se détache de la portion centrale de la ligne âpre.

\section{Epiphyse distale :}

En avant et médialement, l'épiphyse distale présente une surface articulaire en forme de poulie, la trochlée fémorale (articulation en avant avec la patella et distalement avec les plateaux du tibia). De part et d'autre de la trochlée se trouvent les condyles fémoraux latéral et médial. La face médiale du condyle médial correspond à l'épicondyle médial (surmonté par le tubercule de l'adducteur). La face latérale du condyle latéral correspond à l'épicondyle latéral. Sur la face dorsale, les condyles fémoraux sont séparés par la fosse intercondylaire.

\subsection{La patella}


La patella ou rotule est un os situé antérieurement et centralement par rapport à l'épiphyse distale du fémur. Dorsalement, elle présente une surface articulaire pour chaque condyle et une surface pour la fosse intercondylaire.

\subsection{Le tibia}

Le tibia et la fibula sont les os de la jambe. Le tibia supporte le poids du corps et s'articule en haut avec le fémur et en bas avec le talus.

\section{Epiphyse proximale :}

La face ventrale présente des condyles médial et latéral dont la face supérieure plane sert de surface articulaire pour les condyles fémoraux. On parle de plateaux tibiaux médial et latéral. Ces surfaces articulaires sont séparées en avant et en arrière par les aires intercondylaires antérieure et postérieure. Entre ces 2 surfaces se trouve l'éminence intercondylaire qui correspond à la fosse intercondylaire du fémur. Il existe une surface articulaire pour la tête de la fibula au niveau du condyle latéral. On trouve également la tubérosité tibiale (insertion du muscle quadriceps). La face dorsale ne présente pas de relief particulier.

\section{Diaphyse :}

Le corps du tibia a une forme prismatique en coupe transversale. Il présente sur la face ventrale un bord antérieur saillant qui s'élargit vers le haut pour former la tubérosité antérieure du tibia (insertion du ligament patellaire). La face ventro-latérale présente un bord osseux qui permet l'insertion de la membrane interosseuse entre tibia et fibula. La face dorsale est marquée par une ligne oblique = crête oblique du m. soléaire 
Epiphyse distale :

Elle présente médialement un prolongement osseux, la malléole médiale. Elle porte également des facettes articulaires pour le talus et la fibula.

\subsection{La fibula}

La fibula est un os long situé latéralement et en arrière du tibia. Elle participe à la stabilisation de la cheville et constitue un bon greffon osseux.

Epiphyse proximale :

La tête de la fibula présente un prolongement osseux vers le haut, l'apophyse styloïde ou apex de la tête fibulaire. L'épiphyse proximale présente une portion rétrécie, le col de la fibula.

Diaphyse :

Le corps de la fibula est tordu sur son axe et présente 3 bords (antérieur, ventral ou interosseux et postérieur).

Epiphyse distale :

L'épiphyse distale présente un élargissement qui va former la malléole latérale (plus saillante et plus postérieure que la malléole médiale). Elle s'articule avec la surface malléolaire latérale du talus.

6.5 Les os du pied

Le tarse : 
Il comprend 2 parties : le tarse postérieur (avec le talus et le calcanéus) et le tarse antérieur (avec l'os naviculaire, les 3 os cunéiformes et l'os cuboïde).

Tarse postérieur :

- Talus (ou astragale) : il s'articule via la surface articulaire crâniale en haut avec le tibia (et la fibula), en-dessous avec le calcanéus via 2 surfaces articulaires caudales et en avant avec l'os naviculaire. Entre les surfaces articulaires, il existe un petit canal, le sinus du tarse. Il présente une tête, un col et un corps. La tête du talus est dirigée vers l'avant et le côté médial et repose sur le sustentaculum tali du calcanéus. Le corps est de forme arrondie ce qui permet d'accueillir les 2 malléoles latérale et médiale.

- Calcanéus : constitue le talon. Il s'articule par sa face supérieure avec le talus et s'articule par son extrémité antérieure avec l'os cuboïde. Au niveau du bord supérieur de la face médiale du calcanéus se détache une projection osseuse qui soutient la tête du talus : le sustentaculum tali. La face latérale présente une petite crête oblique, la trochlée fibulaire. La tubérosité calcanéenne est l'extrémité postérieure sur laquelle on peut distinguer 3 processus (dont seul le médian prend contact avec le sol en position debout). C'est sur cette tubérosité que va venir s'insérer le tendon d'Achille

Tarse antérieur (depuis le bord médial vers le bord latéral) :

- Os naviculaire : il s'articule en arrière avec la tête du talus et en avant avec les 3 os cunéiformes. Il présente un renflement inférieur de la face médiale, la tubérosité de l'os naviculaire. 
- 3 os cunéiformes : médial, intermédiaire et latéral. Ils s'articulent en arrière avec le naviculaire et en avant avec la base du métatarsien correspondant. Le $3^{\text {ème }}$ cunéiforme s'articule avec l'os cuboïde.

- Os cuboïde : est cubique et présente une tubérosité du cuboïde sur la face inférolatérale. Il s'articule avec le calcanéus en arrière et avec les $4^{\text {ème }}$ et $5^{\text {ème }}$ métatarsiens en avant.

Le métatarse :

Le métatarse est composé de 5 os métatarsiens présentant chacun une base (tête proximale qui s'articule avec les os du tarse antérieur), un corps et une tête distale qui s'articule avec une phalange proximale. Le $1^{\text {er }}$ métatarsien est en contact avec l'os cunéiforme médial, le $2^{\text {ème }}$ métatarsien est en contact avec l'os cunéiforme intermédiaire, le 3ème métatarsien est en contact avec l'os cunéiforme latéral, les $4^{\text {ème }}$ et $5^{\text {ème }}$ métatarsien sont en contact avec l'os cuboïde. Le $5^{\text {ème }}$ métatarsien présente un prolongement le long du bord latéral de l'os cuboïde : le tubercule du $5^{\text {ème }}$ métatarsien. 2 os sésamoïdes sont annexés à la face plantaire de la tête du $1^{\text {er }}$ métatarsien. L'axe du pied passe par le $2^{\text {ème }}$ métatarsien.

Les phalanges :

Chaque orteil comprend 3 phalanges (proximale - moyenne- distale) excepté le gros orteil qui n'en a que 2. Chaque phalange possède un corps et 2 extrémités. 


\section{Les articulations}

Une articulation comprend l'ensemble des structures réalisant la jointure de deux pièces osseuses voisines. Sa finalité est de permettre le mouvement. Il existe des articulations peu mobiles, les synarthroses et des articulations mobiles (synoviales) = les diarthroses.

1. Les synarthroses, articulations peu mobiles

Parmi les synarthroses, il existe :

- Des articulations fibreuses :

- Syndesmose : pont fibreux (pouvant être relativement long) entre 2 os mais qui ne s'ossifie pas (par exemple, la membrane interosseuse qui relie le radius à l'ulna). Il existe un type de syndesmose particulier: la gomphose ou syndesmose dentoalvéolaire qui est un type d'articulation fibreuse où la racine de la dent et sa cavité alvéolaire sont unies.

- Synostose : soudure secondaire entre 2 pièces osseuses. Les os sont séparés par une mince couche de tissus fibreux qui s'ossifie secondairement (ex: sutures crâniennes).

- Des articulations cartilagineuses :

- Synchondrose (ou articulation cartilagineuse primaire): les pièces osseuses sont unies par du cartilage hyalin ou fibrocartilage (ex : plaques épiphysaires).

- Les symphyses, ou les articulations cartilagineuses secondaires sont quant à elles composées de fibrocartilage et permettent peu de mouvements (articulations semi-mobiles) (par exemple, la symphyse pubienne ou disque intervertébral entre 2 vertèbres). 


\section{Les diarthroses}

Elles comportent des surfaces articulaires recouvertes de cartilage hyalin qui sont maintenues en présence par une capsule articulaire. Cette dernière est composée d'une capsule fibreuse et de la membrane synoviale. Elle s'attache aux os à +/- courte distance des surfaces articulaires et délimite la cavité articulaire. La membrane synoviale se trouve sur la face profonde de la capsule articulaire et des surfaces osseuses intra-articulaires dépourvues de cartilage. Cette membrane sécrète le liquide synovial (synovie) servant de lubrifiant. Des ligaments et des muscles vont renforcer la capsule articulaire. Dans certains cas, il existe au niveau de la cavité articulaire un bourrelet fibrocartilagineux (épaule) ou un ménisque (genou) qui s'interpose entre les faces articulaires si les formes ne concordent pas parfaitement.

Il existe 6 différents types d'articulations synoviales en fonction de la forme géométrique des surfaces :

- Sphéroïdes : les surfaces osseuses sont des portions de sphères, l'une pleine l'autre creuse qui peuvent tourner en tous sens l'une sur l'autre. Les mouvements s'exécutent autour de 3 axes se coupant à angle droit. Cela concerne les mouvements d'abduction et d'adduction, de flexion et extension, de rotation médiale et latérale.

- Condylaires : ont deux rayons de courbure différents. L'une est convexe (condyle) et l'autre est concave (cavité glénoïde). Elles sont biaxiales et permettent les mouvements dans 2 plans perpendiculaires entre eux. Cela concerne les mouvements d'abduction et d'adduction, de flexion et extension mais interdise la rotation (ex : articulation radiocarpienne). 
- En selle: deux surfaces en forme de selle inversement conformées s'adaptent et permettent des mouvements dans 2 plans perpendiculaires entre eux. Cela concerne les mouvements d'abduction et d'adduction, de flexion et extension mais interdise la rotation (ex : articulation carpo-métacarpienne).

- Trochléennes : comportent d'un côté une facette en forme de poulie (trochlée) et de l'autre une conformation inverse. Elle permet un seul mouvement perpendiculaire à l'axe longitudinal des os (ex : articulation du coude).

- Trochoïdes : sont des articulations en pivot (cylindre osseux plein pivotant sur son axe dans un cylindre creux généralement ostéo-fibreux. Elle permet les mouvements de rotation (ex : rotation de $\mathrm{C} 1$ autour de $\mathrm{C} 2)$.

- Planes: ont des surfaces articulaires planes et permettent des mouvements de glissement des os l'un sur l'autre (ex : les os du carpe entre eux).

\section{Vascularisation:}

Les articulations sont irriguées par des artères articulaires prenant leur origine sur les vaisseaux péri articulaires. Les artères forment souvent des réseaux péri-artériels. Les veines accompagnent les artères.

\section{Innervation:}

La loi de Hilton stipule que les nerfs qui innervent une articulation sont aussi ceux qui innervent les muscles qui la mobilisent ou la peau qui recouvre leurs sites d'insertion. Les articulations transmettent la notion de proprioception (information responsable de la conscience du mouvement et de la position des différentes parties du corps). Les fibres 
transmettant la douleur sont nombreuses dans la capsule et les ligaments associés. La membrane synoviale est relativement insensible.

\section{Les muscles}

\section{Généralités :}

Les muscles sont des organes dont la propriété principale est la contractilité. Suivant leur constitution histologique, il existe 3 types de tissu musculaire :

- Muscle squelettique : sont fixés directement ou indirectement par des tendons aux os, aux cartilages, aux ligaments, aux fascias ou d'autres structures (œil ou langue). En général, lorsqu'un muscle se contracte, il se raccourcit. L'une de ses extrémités reste fixe donc l'autre se mobilise. Les sites de fixation musculaire sont les insertions. Les muscles squelettiques sont appelés aussi volontaires car on contrôle leur contraction par la volonté pour la plupart. Ils sont appelés aussi striés en raison de l'aspect rayé de leurs fibres au microscope. En histologie, les muscles squelettiques se décrivent par de très longues fibres cylindriques montrant des stries transversales groupées en faisceaux parallèles avec de multiples noyaux.

- Muscle cardiaque : forme la paroi musculaire du cœur, le myocarde. C'est un muscle involontaire. En effet, le rythme cardiaque est contrôlé par un pace maker interne (tissu cardionecteur) qui est lui-même influencé par le SN autonome. Le muscle cardiaque est strié mais il présente une particularité : les disques intercalaires (ou traits scalariformes).

- Muscle lisse: est ainsi nommé en raison de l'absence de striation à l'examen microscopique. Ce sont des muscles involontaires sous contrôle du SNA. On le 
retrouve dans la paroi des vaisseaux (vasoconstriction), du tube digestif (péristaltisme) et de la peau (piloérection).

\section{Le système cardiovasculaire}

\section{Généralités :}

Il existe 3 types de vaisseaux sanguins (artères, veines et capillaires). Le sang quitte le cœur sous haute pression et se distribue au corps au travers d'un système ramifié d'artères. Au bout de ce réseau se trouvent les artérioles qui déversent le sang oxygéné dans des capillaires au niveau desquels les échanges d'oxygène, d'éléments nutritifs et de déchets se font avec le liquide intracellulaire. Le sang qui a traversé le réseau (lit) capillaire passe dans des veinules qui se rassemblent en petites veines puis s'ouvrent dans de plus grosses avant de retourner au cœur.

\section{Les artères}

Les artères sont communément connues pour leur caractéristique de distribution du sang riche en oxygène au corps; en effet, les artères sont plutôt les vaisseaux distrubant le sang de manière centrifuge, à partir du cœur vers l'extérieur : un bel exemple d'artère faisant exception au sang oxygéné est en fait l'artère pulmonaire, qui amène le sang désoxygéné du cœur droit aux poumons. Le sang emprunte des artères dont le calibre est de plus en plus petit. La paroi artérielle est formée de 3 couches : la tunique externe (adventice), moyenne (media) et interne (intima). On distingue 3 types d'artères en fonction de leur épaisseur :

- Artères élastiques : sont les plus volumineuses (aorte et branche de l'arc aortique)

- Artères musculaires : sont des artères de distribution qui contrôlent le flux sanguin vers les différentes parties du corps. 
- Artérioles : sont les plus petites. Elles ont une lumière étroite et une épaisse paroi musculaire.

\section{Les veines}

Elles ramènent le sang des capillaires vers le cœur (circulation centripète, de la périphérie vers le cœur). On distingue 3 types de veines :

- Les veinules : sont les plus petites. Elles se regroupent pour former des plexus veineux.

- Veines satellites : sont généralement double ou multiple et accompagnent les artères profondes. Les veines satellites et l'artère sont entourées d'une gaine vasculaire relativement résistante. En conséquence, pendant la contraction cardiaque, l'artère se contracte et aplatit les veines satellites, ce qui aide à propulser le sang vers le cœur.

- Les veines centrales : veines principales contenant la plupart du sang corporel, vont avoir le rôle de contenir et moduler le volume de sang circulant pour assurer une distribution optimale. Le volume contenu par les grosses veines sera appelé le volume non stressé puisqu'il ne sera pas utilisé directement dans une situation physiologique pour assurer la distribution de nutriments, mais rentrera en jeu dans les cas pathologiques.

4. Les capillaires

Les capillaires sont des conduits qui établissent la connexion entre les versants artériels et veineux de la circulation. Les capillaires sont en réseau interposé entre les artérioles et les veinules. 


\section{Le Cœur}

\subsection{Cycle cardiaque}

Le cœur est divisé en 2 compartiments. Le cœur droit véhicule du sang désoxygéné (envoie le sang vers les poumons pour reoxygénation) et le cœur gauche véhicule du sang oxygéné (envoie le sang vers le reste du corps). Chaque compartiment est divisé en 2 chambres : une qui recueille le sang, l'atrium et une qui le chasse, le ventricule.

- L'atrium gauche est séparé par la valve mitrale (2 cuspides) du ventricule gauche

- Le ventricule gauche est séparé par la valve aortique (3 cuspides) de l'aorte

- L'atrium droit est séparé par la valve tricuspide (3 cuspides) du ventricule droit

- Le ventricule droit est séparé par la valve pulmonaire (3 cuspides) du tronc pulmonaire

Le sang désoxygéné est amené à l'atrium droit par :

- Veine cave supérieure (ramène le sang désoxygéné des régions sus diaphragmatiques) : draine le sang de la tête et du cou (par les veines jugulaires interne - externe - antérieure ainsi que les veines vertébrales) ainsi que les membres supérieurs (par les veines subclavières). Les veines jugulaires interne et subclavière se réunissent pour former les veines brachiocéphaliques droite et gauche. Les deux veines brachiocéphaliques se réunissent pour former la veine cave supérieure. La veine cave supérieure reçoit également le système veineux azygos qui draine le sang veineux de la paroi du tronc.

- Veine cave inférieure (ramène le sang vicié des régions sous diaphragmatiques) : elle nait de la réunion des veines iliaques communes droite et gauche qui naissent de la réunion des veines iliaques externe (pour le membre inférieur) et interne (pour le petit 
bassin). La veine iliaque externe est un prolongement de la veine fémorale à distance du ligament inguinal.

La veine cave inférieure reçoit de nombreux affluents : veines lombaires, veines rénales, veines phréniques inférieures, veine gonadique et surrénale droite (la veine gonadique gauche se jette dans la veine rénale gauche), veines hépatiques (pour le foie qui reçoit la veine porte).

- Sinus coronaire (ramène sang désoxygéné du cœur)

Le sang vicié est donc amené à l'atrium droit. Cycle du cœur droit :

- L'atrium droit se remplit : diastole atriale, correspondant à la systole ventriculaire.

- La valve tricuspide s'ouvre, laissant passer d'abord passivement le sang de l'atrium droit au ventricule droit, puis l'atrium droit se contracte pour pousser ce qui reste: systole atriale, correspondant à la diastole ventriculaire.

- Le sang désoxygéné passe de l'atrium droit au ventricule droit à travers la valve tricuspide qui s'ouvre. Le ventricule droit se remplit: il s'agit de la diastole ventriculaire.

- Une fois le ventricule droit rempli, la valve tricuspide se referme.

- Pendant la systole ventriculaire, le ventricule commence à se contracter pour augmenter sa pression sans que la valve pulmonaire s'ouvre (contraction isovolumétrique), quand la pression ventriculaire dépasse la pression dans le tronc pulmonaire, la valve pulmonaire s'ouvre laissant passer le sang désoxygéné du ventricule droit au tronc pulmonaire et donc aux poumons.

- La valve pulmonaire se ferme. 
- Le tronc pulmonaire se divise en une artère pulmonaire gauche et une artère pulmonaire droite qui vont vers les poumons. Au niveau des alvéoles, le sang se décharge de $\mathrm{CO} 2$ et capte $\mathrm{O} 2$. Le sang est ainsi oxygéné. Le sang oxygéné est amené à l'atrium gauche par 4 veines pulmonaires (deux droites et deux gauches).

Cycle du cœur gauche :

- L'atrium gauche se remplit : diastole atriale, correspondant à la systole ventriculaire.

- La valve mitrale s'ouvre, laissant passer d'abord passivement le sang de l'atrium gauche au ventricule gauche, puis l'atrium gauche se contracte pour pousser ce qui reste : systole atriale (remplissage actif), correspondant à la diastole ventriculaire.

- Le sang oxygéné passe de l'atrium gauche au ventricule gauche à travers la valve mitrale qui s'ouvre. Le ventricule gauche se remplit: il s'agit de la diastole ventriculaire.

- Une fois le ventricule gauche rempli, la valve mitrale se referme.

- Pendant la systole ventriculaire, le ventricule commence à se contracter pour augmenter sa pression sans que la valve aortique s'ouvre (contraction isovolumétrique), quand la pression ventriculaire dépasse la pression dans l'aorte, la valve aortique s'ouvre laissant passer le sang oxygéné du ventricule gauche à l'aorte et donc à tous les organes du corps.

- La valve aortique se ferme.

Circulation artérielle:

L'aorte comprend 3 parties :

- L'aorte ascendante : donne les artères coronaires gauche et droite 
- L'arc de l'aorte : donne

○ Le tronc artériel brachiocéphalique avec l'artère carotide commune droite (qui se divise en carotide externe et interne) et l'artère subclavière droite

$\circ$ L'artère carotide commune gauche

- L'artère subclavière gauche

- L'aorte descendante est séparée par le diaphragme en aorte thoracique et aorte abdominale. L'aorte thoracique donne :

- Des rameaux péricardiques et médiastinaux

- Des rameaux bronchiques

- Des rameaux œsophagiques

○ Les artères intercostales postérieures

- Les artères phréniques supérieures

- Les artères subcostales

L'aorte abdominale donne :

- Des branches pariétales: A phrénique inférieure, A lombaires, A sacrée médiane

○ Des branches viscérales: A surrénalienne moyenne, A rénales, A gonadiques, tronc cœliaque, A mésentérique supérieure et inférieure

L'aorte abdominale se divise ensuite en artère iliaque commune droite et gauche qui se divise en artère iliaque externe et interne. 
5.2. Configuration externe : face antérieure

La face antérieure (ou sterno-costale) est constituée de trois parties :

- Ventriculaire : essentiellement le ventricule droit, un peu le gauche.

- Atriale : surtout l'atrium droit avec l'auricule droit (prolongement antérieur)

- Vasculaire : tronc pulmonaire, aorte, veine cave supérieure

5.3. Configuration externe : face postérieure

La face postérieure est également divisée par le sillon auriculo-ventriculaire en 2 parties : en arrière, se trouve l'oreillette gauche, en avant, les deux ventricules séparés par le sillon interventriculaire qui se prolonge sur la face postérieure du cœur.

\subsection{Configuration externe : base du coeur}

La base est constituée de reliefs musculaires : les muscles pectinés. Elle présente une partie lisse qui rejoint la veine cave supérieure et inférieure : le sinus veineux. On y retrouve l'atrium gauche avec les orifices des quatre veines pulmonaires ainsi que le sulcus terminalis qui sépare le sinus veineux du pectiné.

5.5. Configuration interne du cœur droit

Atrium droit :

- Paroi supérieure : veine cava supérieure + auricule droite

- Paroi inférieure : veine cave inférieure + orifice sinus coronaire

- Paroi latérale : muscles pectines 
- Paroi médiale : fosse ovale

- Paroi antérieure : valve tricuspide

- Paroi postérieure : sinus veineux

\section{Ventricule droit :}

- Présence de la valve tricuspide qui présente trois cuspides (antérieure, postérieure et septale). Les cuspides sont attachées grâce à des cordages tendineux sur des piliers musculaires. Il existe trois piliers (ou muscles papillaires) :

○ Pilier antérieur : est attaché à la paroi antérieure du ventricule et donne des cordages tendineux pour les cuspides antérieure et postérieure.

- Pilier postérieur : donne des cordages tendineux pour les cuspides postérieure et septale.

- Pilier septal : donne des cordages tendineux pour les cuspides antérieures et septale

- Présence de chambres :

- Chambre atriale (ou chambre d'entrée) : où le sang passe de l'atrium au ventricule.

- Chambre artérielle (ou chambre de sortie) : présence de la valve pulmonaire (trois cuspides semi-lunaires, un antérieur et deux postérieurs).

- La bandelette ansiforme : pont musculaire entre la paroi septale et antérieure. C'est à cet endroit que passe le faisceau de His. 
- La crête supraventriculaire ou éperon de Wolf : s'étend depuis la paroi antérieure jusqu'à la paroi septale. Elle sépare la chambre de sortie du reste de la cavité ventriculaire.

5.6. Configuration interne du cœur gauche

Atrium gauche :

- paroi médiale : fosse ovale

- paroi latérale : sans particularité

- paroi postérieure : les quatre veines pulmonaires

- paroi antérieure : valve mitrale

- paroi supérieure : auricule gauche

- paroi inférieure : sans particularité

Ventricule gauche:

- Présence de la valve mitrale constituée de deux cuspides (une antérieure et une postérieure). Deux piliers distincts envoient des cordages vers les deux cuspides :

- Paroi antérieure : présence du pilier antérieur qui envoie des cordages vers les cuspides antérieure et postérieure.

○ Paroi postérieure : présence du pilier postérieur qui envoie des cordages vers les cuspides antérieure et postérieure.

- Présence d'une chambre d'entrée et d'une chambre de sortie. 
- Présence de la valve aortique composée de trois cuspides semi-lunaire (deux antérieures et une postérieure).

5.7. Valves et squelette fibreux du cœur

Le cœur ne s'arrime pas sur un os comme tous les autres muscles. Il s'attache à un squelette fibreux ou cartilagineux qui se situe dans le plan des valves. Les cuspides s'insèrent dans des structures fibreuses $\rightarrow$ les quatre valves sont entourées par quatre anneaux fibreux. Il y a accumulation fibreuse de part et d'autre des valves : les trigones fibreux. Le trigone fibreux gauche est situé entre la valve aortique et mitrale. Le trigone fibreux droit est situé entre la valve mitrale et tricuspide. Celui-ci est traversé par le système cardionecteur que je décrirai juste après. Le squelette fibreux va se continuer dans la paroi de l'aorte, du tronc pulmonaire et dans la partie membraneuse du septum interventriculaire. 


\section{Le système lymphatique}

\section{Généralités}

Le système lymphatique fait partie du système circulatoire. Il s'agit d'un vaste réseau de vaisseaux lymphatiques qui sont reliés à des nœuds lymphatiques (petite masse de tissu lymphoïde). Ce système récole la lymphe qui est un liquide généralement clair et aqueux contenant les mêmes composants que le plasma sanguin (liquide dans lequel les cellules sanguines se trouvent en suspension). Le système lymphatique comprend :

- Les capillaires lymphatiques : très petits vaisseaux qui prennent leur origine dans les espaces intercellulaires de la plupart des tissus.

- Les vaisseaux lymphatiques : vaisseaux collecteurs qui prennent leur origine dans les plexus (réseau de capillaires) lymphatiques et dont les trajets sont jalonnés de nœuds (ganglions) lymphatiques.

- Les nœuds lymphatiques sont traversés par la lymphe dans son trajet vers le système veineux.

- Les lymphocytes circulants qui ont pris dans le tissu lymphoïde (nœuds lymphatiques, rate ou tissu myéloïde de la moelle osseuse rouge).

2. Trajet de la lymphe

Les vaisseaux lymphatiques amènent la lymphe jusqu'au nœud lymphatique. Après avoir traversé un ou plusieurs nœuds lymphatiques, la lymphe pénètre dans de plus gros vaisseaux lymphatiques : les troncs lymphatiques qui se réunissent pour former 2 conduits principaux : 
- Le conduit thoracique : dont la partie initiale se trouve dans l'abdomen et prend la forme d'un sac, la citerne du chyle (citerne de Pecquet). Il monte dans le thorax et puis s'ouvre dans le réseau veineux à la jonction de la veine jugulaire interne et de la veine subclavière gauche (= confluent veineux jugulo-subclavier gauche). Il draine toute la lymphe du corps excepté le quadrant supérieur droit (moitié droite de la tête et du cou, membre supérieur droit, moitié droite du thorax).

- Le conduit lymphatique droit : s'ouvre dans le réseau veineux au niveau du confluent veineux jugulo-subclavier droit. ). Il draine la lymphe du quadrant supérieur droit du corps.

3. Rôles :

- Drainage du liquide tissulaire, récole du liquide interstitiel (espace intercellulaire) et le transport de la lymphe.

- Transport et absorption des graisses: les capillaires lymphatiques spéciaux (les chylifères) récoltent les acides gras à plus de 12C absorbés par l'intestin et transportent le chyle via le conduit thoracique vers le système veineux.

- Réponse immunitaire localisée (infection) : angines, piqure de guêpe 


\section{Le système nerveux}

\section{Généralités}

Le système nerveux permet au corps de réagir à des modifications continues de son environnement extérieur et intérieur. Le système nerveux est divisé structurellement en système nerveux central et périphérique ; fonctionnellement en système nerveux somatique et autonome. Le tissu nerveux comprend 2 grands types de cellules :

- Le neurone : unité structurale et fonctionnelle qui comprend un corps cellulaire (soma) et des prolongements cytoplasmiques: dendrites et axone. La myéline (couche de substances lipidiques et protéiques) forme autour de certains axones une gaine qui accélère la vitesse de conduction de l'influx nerveux. Les neurones communiquent les uns avec les autres par la synapse. La communication se fait au moyen de neurotransmetteurs (agents chimiques libérés par l'un des neurones pouvant stimuler ou inhiber l'autre neurone).

- Les cellules de la névroglie (5 fois plus nombreuses que les neurones) : soutiennent, nourrissent et isolent les neurones.

\section{Système nerveux central}

Il comprend l'encéphale et la moelle épinière. Les rôles principaux sont :

- Intégrer et coordonner les signaux nerveux afférents et efférents.

- Accomplir les plus hautes fonctions neurales telles que la pensée et l'apprentissage.

Les corps cellulaires occupent et constituent la substance grise. Les fibres qui réalisent les interconnexions forment la substance blanche. Au niveau de la moelle épinière, sur une coupe 
transversale, la substance grise apparait représentée par une zone ayant la forme d'un $\mathrm{H}$ entourée de substance blanche. Les extrémités du H sont les cornes : 2 cornes grises dorsales (postérieures) et 2 cornes grises ventrales (antérieures). Il existe également 2 cornes intermédiaires latérales (cornes intermédiolatérales).

3. Système nerveux périphérique

\subsection{Composition}

Il comprend des fibres nerveuses et des corps cellulaires neuronaux situés en dehors du système nerveux central. Il est donc constitué de nerfs qui établissent des connexions entre le système nerveux central et les structures périphériques (muscles etc.).

\subsection{Rôle}

Son rôle est de conduire l'influx nerveux du système nerveux central vers la périphérie ou inversement :

- De la périphérie vers le système nerveux central = fibres afférentes ou sensitives qui transmettent vers le SNC les influx en provenance des organes des sens et des récepteurs sensitifs situés dans différentes parties du corps. Les nerfs sont soit :

- Des nerfs crâniens (originaires de l'encéphale) : 12 paires. Ils quittent la cavité crânienne en franchissant des foramina du crâne.

- Des nerfs spinaux (originaires de la moelle épinière) : 31 paires (8 paires de nerfs cervicaux, 12 paires de thoraciques, 5 paires de lombaires, 5 paires de sacraux et 1 paire de coccygiens). Ils franchissent les foramina intervertébraux de la colonne vertébrale. 
- Du SNC vers la périphérie = fibres efférentes ou motrices qui transmettent l'influx nerveux du SNC vers les organes effecteurs (muscles et glandes).

Structure d'un nerf:

Un nerf est composé de fibres nerveuses qui sont protégées par 3 couches de tissu conjonctif :

- Couche interne $: l^{\prime}$ endonèvre = fine lame de tissu conjonctif qui entoure les axones.

- Couche intermédiaire: la périnèvre = entoure un faisceau de fibres nerveuses périphériques.

- Couche externe : l'épinèvre = épaisse lame de tissu conjonctif qui entoure les faisceaux de fibres. Il contient du tissu graisseux, des vaisseaux sanguins et lymphatiques.

Structure d'un nerf spinal typique:

Un nerf spinal typique prend son origine au niveau de la moelle épinière sous la forme de filets radiculaires qui convergent pour former deux racines nerveuses :

- Racine ventrale qui contient des fibres motrices et dont les corps neuronaux se trouvent dans la corne ventrale de la moelle épinière $\rightarrow$ système efférent.

- Racine dorsale formée de fibres sensitives qui rejoignent la corne dorsale de la moelle épinière $\rightarrow$ système afférent.

Les racines ventrale et dorsale s'unissent pour former un nerf spinal mixte qui se divise immédiatement en deux branches, une ventrale et une dorsale. Les branches dorsales fournissent des fibres nerveuses aux articulations synoviales de la colonne vertébrale, aux muscles profonds du dos et à la peau sus-jacente. Les branches ventrales fournissent des fibres 
nerveuses aux régions antérieure et latérale $d u$ tronc ainsi qu'aux membres supérieur et inférieur.

Composant fonctionnel d'un nerf spinal :

- $\quad$ La voie somatique $=$ fibres somatiques

○ Les fibres somato-afférentes (sensibilité générale) : transmettent les sensations du corps au SNC

- Soit sensibilité extéroceptive: peau (douleur, température, toucher, pression).

- Soit sensibilité proprioceptive : renseigne sur la façon dont le corps est orienté dans l'espace (position des articulations ou tension des muscles et tendons).

○ Les fibres somato-efférentes (somatiques motrices) : transmettent les influx nerveux aux muscles squelettiques.

- La voie viscérale $=$ fibres viscérales

- Les fibres viscéro-afférentes : transmettent les sensibilités douloureuses ou les réflexes en provenance des muqueuses, des glandes et des vaisseaux sanguins.

○ Les fibres viscéro-efférentes : transmettent les influx destinés à la musculature lisse et aux tissus glandulaires.

3.3. Le système nerveux somatique

Le SNS fournit l'innervation sensitive et motrice à toutes les parties du corps sauf les viscères contenus dans le corps, la musculature lisse et les glandes. 


\subsection{Le système nerveux autonome}

Le SNA est également appelé système moteur viscéral. Il comprend des fibres qui innervent la musculature lisse, le muscle cardiaque ou tissu cardionecteur et les glandes. Le SNA est organisé en 2 systèmes: le système sympathique (ou orthosympathique) et le système parasympathique. Dans les 2 systèmes, la transmission de l'influx du SNC jusqu'à l'organe effecteur fait intervenir 2 neurones. Le corps cellulaire du $1^{\text {er }}$ neurone (présynaptique ou préganglionnaire) se trouve dans la substance grise du SNC. Son axone établit un contact avec le corps cellulaire du $2^{\text {ème }}$ neurone (postsynaptique ou postganglionnaire) localisés dans des ganglions autonomes en dehors du SNC. Les fibres postganglionnaires se terminent au niveau de l'organe effecteur. La distinction anatomique entre les 2 systèmes du SNA est basée sur la localisation des corps cellulaires présynaptiques (dans la moelle thoraco-lombaire pour le sympathique et la moelle cranio-sacrée pour le parasympathique). La distinction fonctionnelle entre les 2 systèmes du SNA est pharmacologique (les neurotransmetteurs des neurones présynaptiques sont la noradrénaline pour le sympathique et l'acétylcholine pour le parasympathique).

\subsubsection{Le système nerveux sympathique}

C'est un système catabolique (consomme beaucoup d'énergie) qui se met en route dans une situation de stress ou menaçante (fuite ou lutte par exemple, en anglais fight or flight reaction). Il déclenche un ensemble de réponses qui préparent l'organisme à une activité physique intense :

- Augmentation du rythme cardiaque et de la pression artérielle

- Vasodilatation des artères périphériques 
- Broncho dilatation (passage d'un grand débit d'air)

- Élimination de la chaleur par transpiration

- Adaptation à la vue lointaine : dilatation des pupilles

- Mise au repos de la fonction digestive

- Augmentation de glucose dans le sang

Trajets suivis par les fibres sympathiques:

C'est un système basé sur 2 neurones. Le corps cellulaire du 1er neurone (présynaptique) est situé dans la corne intermédio-latérale de la moelle thoracolombaire (T1-L2). Les corps cellulaires des neurones (postsynaptique) sont rassemblés en ganglions para et prévertébraux. Les ganglions para-vertébraux sont réunis entre eux pour former une chaine sympathique paravertébrale (gauche et droite) tout au long de la colonne vertébrale. Les ganglions pré-vertébraux se trouvent dans les plexus qui entourent les principales branches de l'artère abdominale. La fibre présynaptique va passer par la racine ventrale de la moelle thoraco-lombaire puis par la branche ventrale du nerf spinal pour enfin emprunter via le rameau communicant blanc la chaine ganglionnaire paravertébrale. A ce niveau, il existe 3 possibilités :

- $\quad 1^{\mathrm{er}}$ chemin de base : la synapse avec le neurone postsynaptique se fait dans un ganglion paravertébrale de même niveau. La fibre postsynaptique revient vers les branches ventrale (pour les régions supérieures du corps) ou dorsale (pour les régions inférieur du corps) du nerf spinal via le rameau communicant gris

- $\quad 2 \quad 2^{\text {ème }}$ chemin ascenseur : la fibre présynaptique va au-dessus de TH1 ou en-dessous de L2 et la synapse se fait avec un neurone postsynaptique situé dans un ganglion paravertébral 
plus haut ou plus bas. La fibre postsynaptique revient vers les branches ventrale (pour les régions supérieures du corps) ou dorsale (pour les régions inférieure du corps) du nerf spinal via le rameau communicant gris.

- $\quad 3$ ème chemin viscère :

- Abdomen et pelvis : la fibre présynaptique traverse la chaine ganglionnaire paravertébrale sans y faire de relai. La fibre présynaptique empreinte un nerf splanchnique abdomino-pelvien. La synapse avec le neurone postsynaptique se fait dans un ganglion pré vertébral. La fibre postsynaptique va jusqu' à l'organe.

- Cœur, poumons, œsophage: les fibres postsynaptiques destinées aux viscères thoraciques passent par les nerfs splanchniques cardio-pulmonaire et pénètrent dans les plexus cardiaques pulmonaire et œsophagien.

\subsubsection{Le système nerveux parasympathique}

Le SNP est l'opposé du système sympathique. C'est un système anabolique (préserve l'énergie) qui se met en route dans une situation de repos. Il déclenche un ensemble de réponses qui préparent l'organisme à augmenter la fonction digestive :

- Diminution du rythme cardiaque et de la contraction du cœur

- Vasoconstriction des artères périphériques

- Augmentation du débit de la sphère splanchnique $\rightarrow$ vasodilatation des vaisseaux

- Fonction digestive et rénale active : stimule le péristaltisme et la sécrétion de sucs digestifs ainsi que la miction

- Bronchoconstriction

- Constriction des pupilles 
Trajets suivis par les fibres parasympathiques :

La transmission de l'influx nerveux du SNC jusqu'à l'organe est un système basé sur 2 neurones. Les corps cellulaires des neurones présynaptiques sont situés dans 2 endroits du SNC :

- Au niveau de la substance grise du tronc cérébral: les fibres présynaptiques empruntent les nerfs crâniens III, VII, IX et X , forment le parasympathique crânien.

- Au niveau de la substance grise de la moelle sacrée S2, S3 et S4: les fibres présynaptiques sortent de la racine ventrale er empruntent les nerfs splanchniques pelviens $=$ forment le parasympathique sacral.

L'axone établit un contact avec le corps cellulaire des neurones postsynaptiques localisés pas loin de l'organe cible. Le $1^{\text {er }}$ neurone se situe loin de l'organe cible et le $2^{\text {ème }}$ neurone se situe contre l'organe cible. Dans ce système, la fibre présynaptique est longue et la fibre postsynaptique est courte.

Il y a donc 2 contingents :

- Le parasympathique crânien assure l'innervation parasympathique de la tête et du cou (nerf III, VII et IX) et des viscères thoraciques et abdominaux (nerf vague X) (cœur, poumons et le tube digestif jusqu'au gros intestin, s'arrête à l'angle colique gauche).

- Le parasympathique sacral innerve le colon descendant, sigmoïde et le rectum. 


\section{Le système digestif}

\section{Introduction}

Il existe 4 activités de base de l'appareil digestif : motilité, sécrétion, digestion et absorption. Le tube digestif est ininterrompu de la bouche à l'anus. Il est fait de segments spécialisés.

\section{Cavité buccale, pharynx et œsophage}

Les aliments sont introduits dans la cavité buccale, sont broyés et mélangés à la salive (dont l'amylase amorce la digestion des sucres). La cavité buccale sert de $1{ }^{\text {ère }}$ phase de préparation à la digestion. Le bol alimentaire (aliments mastiqués, salivés et prêts à être avalés) est poussé dans l'arrière-gorge par la langue (volontaire), ce qui déclenche le réflexe de déglutition (volontaire) : fermeture des voies aériennes et propulsion du bol alimentaire dans le pharynx (fermeture du larynx par l'épiglotte). Celui-ci est constitué de 3 parties : nasopharynx (derrière fosse nasale), oropharynx (derrière cavité buccale) et hypopharynx (au-dessus du larynx). La limite anatomique entre le nasopharynx et l'oropharynx est l'axe passant par le palais osseux composé par l'os maxillaire. La limite anatomique entre l'oropharynx et l'hypopharynx est l'os hyoïde. Le bol alimentaire passe de l'oropharynx à l'hypopharynx et envoie le bol alimentaire vers l'œsophage (involontaire). L'œsophage est un organe $(25 \mathrm{~cm})$ reliant l'hypopharynx à l'estomac. Il est situé dans son 1/3 supérieur derrière la trachée et inférieurement derrière le cœur. Il est accolé à la colonne vertébrale mais est séparé par l'aorte. Il est présent dans 3 régions (débute dans la région cervicale, traverse la thoracique et arrive dans la région abdominale). Les 3/4 sont situés dans le thorax. Il présente une musculature lisse qui va faire progresser le bol alimentaire dans l'estomac. 


\section{Estomac}

L'estomac fait suite à l'œsophage. C'est une portion dilatée du tube digestif. C'est une poche située entre l'œsophage et le duodénum. La zone d'abouchement de l'œsophage est le cardia (sphincter, angle de His). En-dessous du cardia se trouve la poche à air (partie de l'estomac où l'air s'accumule). En dessous de la poche à air, se trouve la + grande partie de l'estomac qui est le corps. Le corps se prolonge dans une partie élargie (antre) qui débouche dans une partie coudée (partie pylorique) dont l'extrémité distale sera constituée par un sphincter musculaire puissant le pylore.

- Motilité gastrique :

- Accumule les aliments et les prépare chimiquement (suc) et mécaniquement à la digestion intestinale (contraction)

- Musculature puissante

- Stockage au niveau du corps de l'estomac

- Malaxage du bol alimentaire avec le suc gastrique ( $\mathrm{pH}$ acide) $\rightarrow$ chyme

- Préparation à la digestion enzymatique :

- Des glucides (amylase salivaire)

- Des protéines au niveau de l'antre sous l'effet des sécrétions

- Sécrétion :

- Acide chlorhydrique : dénaturer les protéines, tuer les microbes 
○ Pepsinogène : commencer la digestion des protéines

- Mucus : protéger la muqueuse gastrique de l'autodigestion

○ Facteur intrinsèque: absorption de vitamine B12, production de globules rouges

- Pas d'absorption

\section{Duodénum, foie et pancréas}

Le duodénum mesure 25-30 cm, fixé, accolé derrière lui à l'aorte, veine cave inférieure et les vertèbres. Il présente 4 parties : supérieur I, descendant II, horizontal III et ascendant IV. Il a une forme de cadre dans lequel vient s'encastrer le pancréas afin d'y déverser le suc pancréatique qui contient des enzymes indispensables à la digestion des glucides, protéines et lipides ainsi qu'une solution aqueuse alcaline pour neutraliser l'acidité du chyme. Le pancréas est à la fois une glande exocrine et endocrine. Au niveau de DII, le canal pancréatique de Wirsung et de Santorini (qui s'abouche dans la papille duodénale mineure) avec le canal cholédoque (s'accouplant au niveau de l'ampoule de Vater qui s'abouche dans la papille duodénale majeure), permettant à la bile du foie de se déverser dans le duodénum, vient s'aboucher dans le duodénum. La bile contient les sels biliaires et participe à la digestion et l'absorption des graisses. La vésicule biliaire stocke la bile et libère la bile s'il y a un excès de lipides afin de les digérer. La communication duodéno-jéjunal est l'angle de Treitz.

\section{Intestin grêle}

L'intestin grêle est le principal site de la digestion et absorption. A ce niveau, on assiste à la fin de la digestion des glucides et des protéines. La digestion des lipides a lieu totalement dans la 
lumière de l'intestin. L'intestin grêle se termine dans le gros intestin au niveau du caecum. Il présente 3 parties : duodénum (fixe), jéjunum (mobile) et iléon ou ileum (mobile).

\section{Duodénum :}

Voir point 4 .

Jéjuno-iléon :

Le jéjunum (5-8m) a une disposition en anses horizontales tandis que l'iléum a une disposition en anses verticales. C'est l'organe de l'absorption des glucides, protéines et lipides grâce à une muqueuse qui est plissée pour augmenter la surface d'absorption (anses grêles). La caractéristique essentielle est la mobilité grâce à un ligament (péritoine) qui rattache l'intestin grêle à la paroi postérieure. La fin du jéjunum se situe au niveau de la valve iléo-caecale qui correspond à l'abouchement de l'iléon dans le caecum. L'iléon montre moins de plis mais présente des amas lymphoïdes (plaques de Peyer). Il est peu vascularisé.

\section{Gros intestin}

Le gros intestin fait suite au jéjuno-iléon. Il est de diamètre plus grand et présente des haustrations (plis semi lunaires au niveau de la muqueuse). Il existe 3 ténias colis qui sont des fibres musculaires lisses longitudinales avec des morceaux de graisses attachés (appendices épiploiques). Il est le site de concentration des résidus non digérés de l'alimentation et des déchets biliaires. Il a un rôle d'absorption d'eau et d'électrolytes et effectue des mouvements de masses jusqu'au rectum (réflexe de défécation). Il présente 3 parties : caecum et appendice, colon, rectum. La limite entre le colon et le rectum est la fin des ténias colis = région rectosigmoïdienne. Le caecum et appendice sont situés sous l'abouchement de la valve iléo-caecale. 
Les ténias colis débutent où s'insère l'appendice vermiforme. La différence entre le caecum et le colon est que le caecum se trouve en dessous de l'abouchement de l'ilion terminal (pas de différence anatomique). Le colon a une structure en cadre et se divise en 4 : colon ascendant, transverse, descendant et sigmoïde qui se prolonge dans le rectum. Le rectum ressemble à une ampoule. Il accumule les selles (2 sphincters strié et lisse). Il se termine par le canal anal (colonnes anales pour sécrétion de graisse) qui s'abouche à la peau au niveau de l'anus.

\section{Subdivisions de la cavité abdominale}

Afin de localiser les organes abdominaux ainsi que les douleurs, les cliniciens distinguent 9 régions qui sont délimitées par 4 plans :

- 2 plans horizontaux :

○ Plan subcostal : bord inférieur du $10^{\text {ème }}$ cartilage costal

- Plan intertuberculaire: passant par les tubercules iliaques (partie la plus latérale de la crête iliaque)

- 2 plans verticaux : les plans médio-claviculaires passant par le milieu des clavicules et par le milieu des ligaments inguinaux.

\begin{tabular}{|l|l|l|}
\hline Hypochondre D & Épigastre & Hypochondre G \\
\hline Flanc D & Région ombilicale & Flanc G \\
\hline Fosse iliaque D & Hypogastre & Fosse iliaque G \\
\hline
\end{tabular}

8. Le péritoine. 


\section{Définition :}

Le péritoine est une membrane séreuse qui tapisse la paroi de la cavité abdominale et pelvienne. Il se réfléchit à partir de la paroi vers divers organes dont il recouvre les surfaces. Le péritoine qui tapisse les parois est appelé péritoine pariétal tandis que le péritoine qui recouvre les organes est appelé péritoine viscéral. On reconnait donc au péritoine un feuillet pariétal (tapissant les parois abdomino-pelviennes), un feuillet viscéral (revêtement séreux des organes) et des replis membraneux qui relient le péritoine pariétal au péritoine viscéral. Ces replis permettant la continuité péritonéale engainent les pédicules vasculo-nerveux qui vont de la paroi aux organes.

Il existe différents types de replis :

- Les méso : sont des doubles feuillets péritonéaux unissant des portions du tube digestif à la paroi abdominale en leur gardant une mobilité +/- importante (ex : le mésogastre en connexion avec l'estomac, mésentère en connexion avec le jéjuno-iléon).

- Les ligaments : sont des replis formés de 2 feuillets péritonéaux reliant un organe digestif plein (foie, rate) ou un organe génito-urinaire à la paroi ou d'un organe digestif à un autre.

- Les épiploons ou omentums : sont des feuillets péritonéaux doubles reliant l'estomac à d'autres viscères.

- Grand omentum: débute au niveau de la grande courbure de l'estomac, descend devant les anses intestinales puis se replie sur lui-même vers l'arrière pour s'attacher au colon transverse.

- Petit omentum : va de la petite courbure de l'estomac et DI au foie. 
La cavité péritonéale:

Le péritoine pariétal et viscéral, les mésos, les ligaments et épiploons sont des parties d'une même membrane continue qui limite une cavité virtuelle, la cavité péritonéale. On la divise en 2 parties inégales :

- La grande cavité péritonéale : va du diaphragme au pelvis. Elle est elle-même divisée en 2 par le colon transverse et son méso.

○ Étage sus-mésocolique : entre le diaphragme et le mésocolon transverse. Il comprend les espaces situés entre le foie, la face ventrale de l'estomac, la rate et DI.

- Étage sous-mésocolique : entre le mésocolon transverse (face caudale) et le plancher pelvien. Il est cloisonné par le mésentère, le mésosigmoide et le ligament large de l'utérus. Il est encadré par les colons ascendant et descendant.

- La bourse omentale (ou arrière cavité des épiploons) : est située en arrière de l'estomac et en avant du péritoine pariétal et du pancréas. Elle est limitée à gauche par la rate.

J'ai eu l'occasion d'approfondir mes connaissances en anatomie à partir de ma troisième année de bachelier en médecine, où j'ai été nommé assistant en anatomie. Il est vrai que lorsqu'on enseigne, en réalité on apprend à soi-même. 
Ce syllabus est dédié à tous ceux qui, comme moi, trouveront dans l'étude de l'anatomie humaine une perfection sans égal parmi les autres sciences.

Giovanni Briganti 